\title{
Quarks, Gluons, Colour: Facts or Fiction?
}

\author{
Detlev Buchholz \\ II. Institut für Theoretische Physik, Universität Hamburg \\ Luruper Chaussee 149, D-22761 Hamburg, Germany
}

\begin{abstract}
A general method is presented which allows one to determine from the local gauge invariant observables of a quantum field theory the underlying particle and symmetry structures appearing at the lower (ultraviolet) end of the spatio-temporal scale. Particles which are confined to small scales, i.e., do not appear in the physical spectrum, can be uncovered in this way without taking recourse to gauge fields or indefinite metric spaces. In this way notions such as quark, gluon, colour symmetry and confinement acquire a new and intrinsic meaning which is stable under gauge or duality transformations. The method is illustrated by the example of the Schwinger model.
\end{abstract}

\section{Introduction}

The structure of hadronic matter at small spatio-temporal scales is commonly interpreted in terms of charged particle-like structures (quarks, gluons) which do not appear at large scales because of confinement. The origin of this interpretation is (a) the quark model, which explains the form of the hadronic spectrum, (b) the phenomenological parton picture of deep inelastic collision processes and (c) the perturbative treatment of quantum chromodynamics, where the particle interpretation enters through the use of Feynman rules for the gauge and matter fields.

In view of recent interesting developments in gauge theory (cf. [1] for a review) one may, however, be tempted to question the basis for such an interpretation. For in a large class of four dimensional (supersymmetric) gauge theories one can proceed by duality transformations from a description of the observables in terms of gauge fields of "electric type" to a "magnetic" description and viceversa. By these transformations one changes also the gauge group and the number of gauge particles, converts strongly coupled theories into weakly coupled ones, confinement into screening etc. So it seems that none of these concepts has an intrinsic meaning. As N. Seiberg has put it [1]: Gauge symmetries might not be fundamental. They might only appear as long distance artifacts of our description of the theory. 
We do not subscribe to this conclusion, but there is a lesson to be drawn from these examples which, as a matter of fact, is not entirely new: it makes no sense to attribute a physical interpretation to the unobservable gauge fields of a theory. Even the statement that some observable can be represented by a combination of certain gauge fields may be physically meaningless. There may be another representation of this observable in terms of other fields in another formulation of the theory. Thus, if it comes to the physical interpretation of a theory, the observables have to speak for themselves. (We mention as an aside that this point of view has been advocated in the algebraic approach to quantum field theory for more than three decades [2].) In particular, the question of whether quarks, gluons and colour are to be regarded as elements of physical reality has to be decided on the basis of the observables, that is, without recourse to gauge fields.

If one adopts this point of view, many theories seem to lose the basis for their interpretation, a prominent example being massless quantum electrodynamics in two dimensions (Schwinger model). It is well known that the local observables in this model can be represented as functions of the free neutral massive scalar field [3]. The absence of charged particles from the physical sector has been interpreted as confinement [4] or screening [5], to mention only two articles. But if one forgets about the way how the theory has been constructed and looks only at the final result, the observables, there seems to be no basis for either one of these interpretations. For one could have started just as well with a free field Lagrangian and would have arrived at the same set of observables. Similar examples of local gauge theories which can be reinterpreted in terms of non-gauge theories exist also in three dimensions [6].

It is the aim of the present article to show that in spite of these ambiguities one can decide in a precise and intrinsic manner whether or not a theory describes at small scales a structure corresponding to the physical idea of confined quarks and gluons, carrying a colour charge. The particles and charges appearing in our approach at the lower (ultraviolet) end of the spatio-temporal scale do not require a description in terms of unphysical degrees of freedom in some gauge theory. As we shall see, they can be described as physical states and symmetries of the algebra generated by the observables at arbitrarily small scales. In order to avoid from the outset any confusion with standard terminology we call these entities ultraparticles [7] and ultrasymmetries.

The basic idea of our approach is to apply a new type of renormalization group (scaling) transformation [8] to the local observables of the theory and to proceed to the scaling limit. This method is outlined in Sec.2. The resulting scaling limit theory has in general a different particle content and different symmetries. These properties can be deduced from the corresponding observables by algebraic methods developed in [9]. The main results of that work are also briefly reviewed in Sec.2. In accordance with the construction, the particle content and the symmetry group of the scaling limit theory are regarded as the ultraparticles and ultrasymmetries of the underlying theory. Some relevant possibilities for these structures are discussed in Sec.3, where also a physically meaningful criterion for confinement of ultraparticles is stated. In Sec.4 the general method 
is illustrated on the example of the Schwinger model which turns out to have a non-trivial ultraparticle and ultrasymmetry structure. The article closes with a brief summary of the general method.

\section{Ultraparticles and ultrasymmetries}

Given a theory by specification of a Lagrangian, say, one is faced with two basic problems. First one has to perform the construction of the theory and then to provide the physical interpretation of the mathematical results. We are dealing here with the latter problem and therefore may assume that on the constructive side everything has been said and done: all correlation functions (vacuum expectation values) of the local observables have been computed and - if necessary continued from Euclidean to Minkowski space points. Applying the reconstruction theorem $[2,10]$, one can then represent the observable fields, currents, stress energy tensor etc. as operators on a physical Hilbert space. Our problem is thus to extract from these data the physical interpretation of the theory at very small scales.

Let $\phi(x)$ be a (hermitean) operator representing an observable at the spacetime point $x{ }^{1}$ Since we are interested in the interpretation of the theory at small scales we have to study the effect of a change of the spatio-temporal scale on the observables, say $\phi$, while keeping the scales $c$ of velocity and $\hbar$ of action fixed. According to the basic ideas of renormalization group theory, (cf. [11] and references quoted there), the observables corresponding to $\phi$ at other scales are obtained by setting

$$
\phi_{\lambda}(x) \doteq N_{\lambda} \phi(\lambda x), x \in \mathbb{R}^{4},
$$

where $\lambda>0$ is a scaling factor. We call $\phi_{\lambda}$ the observable at scale $\lambda$. Whereas the action of the scaling transformation on the argument of $\phi$ needs no explanation, its effect on the scale of $\phi$, given by the positive factor $N_{\lambda}$, is more subtle. The familiar idea is to adjust this factor in such a way that the expectation values of the observables at scale $\lambda$ have the same order of magnitude for all $\lambda>0$. The precise way in which this idea is implemented is a matter of convention. One may pick for example a suitable (real) test function $f$ and consider

$$
\phi_{\lambda}(f) \doteq \int d^{4} x f(x) \phi_{\lambda}(x) .
$$

The factor $N_{\lambda}$ can then be fixed by demanding that

$$
\left\langle V\left|\phi_{\lambda}(f) \phi_{\lambda}(f)\right| V\right\rangle=\mathrm{const} \text { for } \lambda>0,
$$

where $|V\rangle$ denotes the vacuum vector. But one could impose this constraint just as well on some higher moment of $\phi_{\lambda}(f)$, giving a somewhat different result for

\footnotetext{
${ }^{1}$ Strictly speaking, $\phi(x)$ is an operator valued distribution, i.e., becomes an operator only after integration with a suitable test function. Tensor indices etc. will be suppressed.
} 
$N_{\lambda}$ in general. We denote the factor $N_{\lambda}$ which has been fixed by imposing such a renormalization condition by $Z_{\lambda}$.

If one wants to proceed to the scaling limit $\lambda \rightarrow 0$ there appears, however, a well-known problem. The product of operators at neighbouring spacetime points is quite singular, so the factors $Z_{\lambda}$ tend to 0 as $\lambda \rightarrow 0$. One needs rather precise information on the short distance properties of the correlation functions and the way how $Z_{\lambda}$ approaches 0 in order to be able to control the scaling limit.

This problem can be solved under favourable circumstances (asymptotically free theories) since renormalization group equations and perturbative methods provide reliable information. The approach works also in certain renormalizable theories where the underlying renormalization group equations have a nonvanishing but small ultraviolet fixed point. Yet in the case of theories without a (small) ultraviolet fixed point the method does not lead to reliable results, nor can a rigorous treatment of non-renormalizable theories be even addressed. Moreover, within the present context this method is also not appropriate because of conceptual reasons. For renormalization group equations are frequently formulated in terms of non-observable fields which we want to omit from our discussion. Thus, at first sight, a general definition of the scaling limit of the observables of a theory would seem impossible.

There is, however, a solution to this problem which is surprisingly simple [8]. Within the present setting ${ }^{2}$ it can be described as follows. In a first step one proceeds from the unbounded operators $\phi_{\lambda}(f)$ to corresponding bounded operators, such as the unitary (generating) operators $\exp \left(i \phi_{\lambda}(f)\right)$. This has the effect that, irrespective of the choice of the factor $N_{\lambda}$ in the defining equation (2.1) of $\phi_{\lambda}$, there do not appear any divergence problems. The resulting operators are bounded in norm, uniformly in $\lambda>0$.

The second step is to restrict the four-momentum of these bounded operators in accord with the uncertainty principle. Roughly speaking, one considers only observables at scale $\lambda$ which can transfer to physical states four-momentum proportional to $\lambda^{-1}$. Thus these observables occupy, for all $\lambda>0$, a fixed phase space volume. The desired operators are obtained by spacetime averages,

$$
A_{\lambda} \doteq \int d^{4} y g(y) \exp \left(i \phi_{\lambda}\left(f_{y}\right)\right), \quad \lambda>0,
$$

where $g$ is any test function and $f_{y}(x) \doteq f(x-y)$.

The results in [8] imply that this restriction on the four-momentum transfer has in general the following effect: if one chooses $N_{\lambda}$ in (2.1) such that the quotient $N_{\lambda} / Z_{\lambda}$ tends to infinity in the limit of small $\lambda$, then all correlation functions involving the corresponding sequence of operators $A_{\lambda}$ converge to 0 . Similarly, if $N_{\lambda} / Z_{\lambda}$ tends to 0 , then $A_{\lambda}$ converges (in the sense of correlation functions) to $\int d^{4} x g(x) \cdot 1$. Thus in either case the operators $A_{\lambda}$ tend in the scaling limit to multiples of the identity. Only in the special case where the asymptotic behaviour of $N_{\lambda}$ coincides with that of $Z_{\lambda}$ does it normally happen

${ }^{2}$ The framework in [8] is more general. It applies to arbitrary finitely localized observables, including Wilson loops, finite strings etc. 
that the correlation functions of the operators $A_{\lambda}$ retain a non-trivial operator content in the scaling limit.

In view of this fact one need not know the behaviour of $Z_{\lambda}$ and may admit in the above construction all possible factors $N_{\lambda}$. The theory takes care by itself of those choices which are unreasonable and lets them disappear (become trivial) in the scaling limit. It is only if $N_{\lambda}$ has the "right" asymptotic behaviour that the sequences $A_{\lambda}$ give rise to non-trivial operator limits. One may view this method as an implicit way of introducing renormalization group transformations. It allows one to study the short distance properties of local observables in an intrinsic, model independent manner. Actually, this method is also useful in concrete computations, as we shall see in Sec.4.

It is mathematically convenient to regard the operators $A_{\lambda}$, obtained by the above procedure, as operator valued functions of the scaling parameter $\lambda>0$. These functions form in an obvious way an algebra $^{3}$, called scaling algebra in [8]. For the construction of the scaling limit of the observables one considers all expectation values of sums and products of these operator functions in the limit $\lambda \rightarrow 0$,

$$
\lim _{\lambda \rightarrow 0}\left\langle V\left|\sum A_{\lambda} A_{\lambda}^{\prime} \cdots A_{\lambda}^{\prime \prime}\right| V\right\rangle=\left\langle V_{0}\left|\sum A_{0} A_{0}^{\prime} \cdots A_{0}^{\prime \prime}\right| V_{0}\right\rangle .
$$

Actually, these limits may only exist for suitable subsequences of the scaling parameter $\lambda$. We disregard this problem here and refer to the thorough discussion of this point in [8], cf. also the remarks in Sec.4. What is of interest here is the fact [8] that the limits of these expectation values determine, by an application of the reconstruction theorem to the scaling algebra, a local, Poincaré covariant theory with vacuum vector $\left|V_{0}\right\rangle$. This explains the notation on the right hand side of relation (2.5). Disregarding certain exceptional cases (cf. the classification of scaling limits in [8]) one can represent the operators $A_{0}$ in this expression in the form

$$
A_{0}=\int d^{4} y g(y) \exp \left(i \text { const } \phi_{0}\left(f_{y}\right)\right)
$$

where $\phi_{0}$ is the scaling limit of $\phi_{\lambda}$ and the value of the constant depends on the choice of the normalization factors $N_{\lambda}$. Thus, by this universal method, one can define the scaling limit of the observables in any given theory.

We emphasize that the operators $A_{0}$ will in general have properties which are very different from those of the original observables. They are the observables in a "new theory", the scaling limit theory, acting on their own "new Hilbert space". To a certain extent the situation may be compared with collision theory, where one proceeds by the LSZ-limit from interacting fields to free asymptotic fields. But whereas the asymptotic fields describe the same global (unbroken) symmetries and particle spectrum as the underlying interacting fields, these features may change if one proceeds to the scaling limit. As we shall see, it is this very fact which allows one to describe the phenomenon of confinement.

\footnotetext{
${ }^{3}$ Sums, products and hermitean conjugates are pointwise defined, $(A+B)_{\lambda} \doteq A_{\lambda}+B_{\lambda}$, $(A \cdot B)_{\lambda} \doteq A_{\lambda} \cdot B_{\lambda},\left(A^{*}\right)_{\lambda} \doteq(A)_{\lambda}^{*}$. There exists also a norm on this algebra [8].
} 
The determination of the particle spectrum and the global symmetries of the scaling limit theory requires, however, some further steps. So far we know (in principle) only the vacuum expectation values of all observables in the scaling limit theory. Thus the Hilbert space obtained from these data by the reconstruction theorem contains only neutral states. There may be also charged states in the scaling limit theory. How does one find them?

This question can be compared to the following familiar problem in group theory. Consider a group, such as $S L(2, \mathbb{C})$, in its defining representation and regard this representation as a faithful picture of some "abstract group", defined by its multiplication table. How does one find the other representations of this abstract group? There is no easy general answer to this question, but there are general strategies which eventually lead to a solution.

The situation is very similar in our field theoretic problem. Instead of dealing with a group we are dealing with an algebra $\mathcal{A}_{0}$ which is generated by all sums and products of the local observables $A_{0}$ in the scaling limit theory and acts on the Hilbert space of neutral scaling limit states. We regard $\mathcal{A}_{0}$ as a faithful representation of some "abstract algebra", denoted by the same symbol, which is defined through the algebraic relations in $\mathcal{A}_{0}$ (such as commutation relations, fusion rules, equations of motion etc.). If there exist charged physical states in the scaling limit theory they correspond to vectors in other (disjoint) Hilbert space representations of $\mathcal{A}_{0}$. Thus the problem at hand is to determine these representations.

In analogy to the case of non-compact groups, the algebra $\mathcal{A}_{0}$ has in general a tremendous number of representations, corresponding to very different physical situations (states). Some of them may be very interesting, such as thermal equilibrium states, but they are of no relevance in the present context. Others may be regarded from the outset as pathological, such as states with a rapidly increasing energy density at infinity. Thus, what is required are certain a priori conditions which characterize states describing (charged) elementary systems. Such selection criteria have been formulated in the literature in mathematically precise terms, cf. for example [2]. It suffices therefore to state them here in a somewhat informal way.

The first condition which we impose on the representations of $\mathcal{A}_{0}$ to be considered here is the requirement that energy-momentum operators can be defined on the respective Hilbert spaces and satisfy the relativistic spectrum condition (positivity of energy in all Lorentz frames). Note that $\mathcal{A}_{0}$ contains only observables which are localized in bounded spacetime regions. Since the energy-momentum operators are obtained by integrating the stress energy tensor over all of space this condition imposes a non-trivial constraint.

The second condition implements the idea that elementary systems are localized excitations of the vacuum. In order to test whether a vector $\left|E_{0}\right\rangle$ describes such an excitation one has to compare the expectation values $\left\langle E_{0}\left|A_{0}\right| E_{0}\right\rangle$ and the vacuum expectation values $\left\langle V_{0}\left|A_{0}\right| V_{0}\right\rangle$. These expectation values should coincide for all observables $A_{0}$ which are localized in the spacelike complement of any sufficiently large region, containing the localization region of $\left|E_{0}\right\rangle$. Note that the 
localization properties of $A_{0}$ can be prescribed by choosing in relation (2.4) test functions with specific support properties.

The two conditions in this selection criterion have a clear-cut physical interpretation, but they provide only a rather implicit description of the relevant set of states and representations. It is therefore natural to ask whether there is a more explicit characterization. An answer to this question is given by the following deep result of Doplicher and Roberts [9] which we formulate here for the algebra of observables in the scaling limit: (a) the algebra $\mathcal{A}_{0}$ can be extended to some field algebra $\mathcal{F}^{0}, \mathcal{A}_{0} \subset \mathcal{F}^{0}$, which is generated by charge carrying Bose respectively Fermi fields. (b) There is a compact group $G_{0}$ which acts on $\mathcal{F}^{0}$ by automorphisms, that is the basic fields in $\mathcal{F}^{0}$ transform like vectors in irreducible unitary representations of $G_{0}$. Moreover, the elements of $\mathcal{A}_{0}$ are exactly the fixed points in $\mathcal{F}^{0}$ under the action of $G_{0}$. (c) The Hilbert space spanned by all state vectors $\left|E_{0}\right\rangle$, described in the selection criterion, is obtained by applying all elements of $\mathcal{F}^{0}$ to the vacuum vector $\left|V_{0}\right\rangle$. (d) The algebra $\mathcal{F}^{0}$ and the group $G_{0}$ are uniquely fixed by these properties.

This result is of importance for the problem at hand. For it shows that one can determine from the scaling limit algebra $\mathcal{A}_{0}$ on the Hilbert space of neutral states the global gauge group $G_{0}$ of the scaling limit theory. These are the symmetries appearing in the original theory at very small scales, so they are the ultrasymmetries. Similarly, by analyzing the discrete (atomic) spectrum of the mass operator on the Hilbert space spanned by the set of vectors $\mathcal{F}^{0}\left|V_{0}\right\rangle$ one can determine the set $\Sigma_{0}$ of charged and neutral particles in the scaling limit theory. These are the ultraparticles. In view of the canonical construction of the scaling limit and point (d) mentioned above, both, the ultraparticles and the ultrasymmetries are intrinsic features of the underlying theory.

By the same general method one can recover from the algebra $\mathcal{A}$, which is generated by the observables in the underlying theory, the global symmetry group $G$, the algebra of charge carrying fields $\mathcal{F}$ and the physical particle spectrum $\Sigma$.

We emphasize that these structures are fixed by the class of states which one has selected. Yet the conditions given above may sometimes be too restrictive. If, for example, there appear particles in the theory carrying a gauge or quantum topological charge in the sense of [12], one has to consider also string-like excitations of the vacuum which are localizable in arbitrary spacelike cones. In theories where all physical particles are massive, these are the worst possible localization properties of elementary states [12]. We note that the results of Doplicher and Roberts can be generalized to this extended class of states [9]. If one wants to consider also particles carrying electric charge one has to proceed to an even larger class of states [13] and can no longer rely on the characterization of particle states by discrete contributions in the mass spectrum (infraparticle problem) [14].

These more general cases arise whenever a particle carrying a gauge charge appears in the physical spectrum, i.e., is not confined. But then there are no problems with its physical interpretation. We will therefore restrict our attention here to the simple case where all states of interest on $\mathcal{A}$ and $\mathcal{A}_{0}$ are well-localized, the corresponding field algebras $\mathcal{F}$ and $\mathcal{F}^{0}$ are hence generated by local field 
operators, and particles correspond to sharp eigenvalues of the mass operator. The extension of our method to the more general situation indicated above is outlined in Sec.4 on the example of the Schwinger model.

Before we turn to the discussion of the physical interpretation of these structures let us comment on the problem of computing in a given theory the states and representations of interest, described above. As in the case of group theory, there are no simple general solutions, but there are strategies.

The first approach is based on the observation that the desired state vectors $\left|E_{0}\right\rangle$ give rise to states (expectation functionals) $\mathcal{E}_{0}(\cdot) \doteq\left\langle E_{0}|\cdot| E_{0}\right\rangle$ on the algebra $\mathcal{A}_{0}$ with certain characteristic properties. Conversely, given a state $\mathcal{E}_{0}(\cdot)$ with such properties, one can reconstruct the corresponding vector $\left|E_{0}\right\rangle$ and representation of $\mathcal{A}_{0}$ by the reconstruction theorem. Making use of the results of Doplicher and Roberts mentioned above one knows from the outset that the states $\left\langle E_{0}|\cdot| E_{0}\right\rangle$ can be approximated by suitable sequences of neutral states. Roughly speaking, one has to exhibit vectors describing bilocalized excitations of the vacuum state, where in one region about the spacetime point 0 , say, sits a charge and in another region about the point $x$ some compensating charge, $\left|E_{0} \times \bar{E}_{0}(x)\right\rangle$ in symbolic notation. These vectors lie in the given Hilbert space of neutral states. One then shifts $x$ to spacelike infinity with the result that the compensating charge has no longer any effect on the local observables,

$$
\lim _{x}\left\langle E_{0} \times \bar{E}_{0}(x)\left|A_{0}\right| E_{0} \times \bar{E}_{0}(x)\right\rangle=\left\langle E_{0}\left|A_{0}\right| E_{0}\right\rangle, A_{0} \in \mathcal{A}_{0}
$$

Thus, by shifting a compensating charge "behind the moon" [15], one can proceed from neutral states to charged states and leave the Hilbert space. The trick is to require convergence only in the weak sense of relation (2.7).

The second approach works only in very special cases which may, however, be of particular interest in physics. Namely, let the given theory describe states and observables which, at small scales, behave as if there is no interaction (in a loose sense, there is "asymptotic freedom"). This feature should imply that the algebra $\mathcal{F}^{0}$ is generated by free fields and $\mathcal{A}_{0}$ contains the $G_{0}$-invariant combinations of these fields. Moreover, basic observables in $\mathcal{A}_{0}$ such as the scaling limits of observable currents, the stress energy tensor etc. should be bilinear expressions in these free fields. But from such operators one can reconstruct the underlying fields directly by a simple algebraic method invented in [16].

\section{Structure of the scaling limit}

We have established in the preceding section a general method for the analysis and interpretation of a given theory at arbitrarily small scales. Starting from the algebra $\mathcal{A}$ of local observables one first proceeds to the corresponding algebra $\mathcal{A}_{0}$ in the scaling limit theory and then determines the associated field algebra $\mathcal{F}^{0}$, the group $G_{0}$ of ultrasymmetries and the set $\Sigma_{0}$ of ultraparticles. The structure of $\mathcal{F}^{0}, G_{0}, \Sigma_{0}$ depends on detailed properties of the underlying theory and has to be computed in each case. 
In analogy to the case of collision theory, where the interpretation of the theory is accomplished by comparing the algebras of incoming and outgoing fields which are related by the scattering matrix, some interesting dynamical information is obtained by comparing the algebras $\mathcal{A}$ and $\mathcal{A}_{0}$, respectively the corresponding data $\mathcal{F}, G, \Sigma$ and $\mathcal{F}^{0}, G_{0}, \Sigma_{0}$. We discuss in the following some relevant cases.

A first important possibility is the case $G_{0} \supset G$. It appears if there is an enhancement of symmetries at small scales, as it is expected in some asymptotically free gauge theories. But we emphasize that one cannot decide on the basis of the observables $\mathcal{A}_{0}$ whether the extra symmetries appearing in $G_{0}$ are related to some underlying local gauge group. For these observables test the properties of the underlying theory in an infinitesimal neighbourhood of a given spacetime point. But, as is intuitively clear, in such a "neighbourhood" one cannot distinguish between local and global gauge transformations.

Another interesting possibility is $G_{0} \subset G$ or that there do not appear any ultrasymmetries at all in the scaling limit. The latter case may be met for example in theories without ultraviolet fixed point, should they exist in some sense as continuum theories [17]. One would expect in such theories that no choice of the normalization factor $N_{\lambda}$ in relation (2.1) leads to non-trivial operators in the scaling limit. Then the algebra $\mathcal{A}_{0}$ consists of multiples of the identity and $G_{0}$ is trivial. (According to the terminology of [8] the scaling limit would be "classical".)

There may be also cases where the relation between $G$ and $G_{0}$ is more complicated. Parts of the symmetries in $G$ may disappear in the scaling limit by a mechanism as just described and new symmetries may appear. Then $G$ and $G_{0}$ have at best some subgroup in common.

Let us now turn to the particle aspects in the scaling limit. Here we confine ourselves to the most interesting case of dilation-invariant scaling limit theories. They arise if the limits in (2.5) are unique, cf. the corresponding results in [8]. In case of dilation invariance all ultraparticles in $\Sigma_{0}$ are massless. Moreover, the ultraparticles do not interact at very small scales, that is the respective incoming and outgoing collision states, determined by standard collision theory from the fields in $\mathcal{F}^{0}$, coincide [18].

Of particular interest is the special case where the scaling limit theory has a complete ultraparticle interpretation ("asymptotic completeness" in the ultraviolet). Then $\mathcal{F}^{0}$ describes a set $\Sigma_{0}$ of non-interacting massless particles and consequently should be generated by massless free fields. ${ }^{4}$ We say in this case the underlying theory is a free theory at small scales. (It seems likely that this definition agrees with the notion of asymptotic freedom in renormalization group theory [11].) Thus we arrive at the following conclusion:

A theory has a complete ultraparticle interpretation if and only if it is a free theory at small scales.

\footnotetext{
${ }^{4}$ In [19] this fact has been established for the case where $\mathcal{F}^{0}$ is generated by some local scalar field, cf. also [20] and [21]. But the methods can be extended to more general situations.
} 
The extreme opposite case is present if $\Sigma_{0}$ is empty but $\mathcal{F}^{0}$ is non-trivial. Then the observations made at small scales have to be interpreted without reference to a particle picture. This situation is expected to occur in theories with a non-trivial ultraviolet fixed point. There may be also intermediate cases where $\Sigma_{0}$ is non-empty, but there are vectors in the space $\mathcal{F}^{0}\left|V_{0}\right\rangle$ which do not have a particle interpretation. In any case, the structure of $\Sigma_{0}$ is - in the same way as that of $G_{0}-$ an observable feature of the underlying theory.

Let us finally turn to the issue of confinement of ultraparticles. We want to cast the physical idea, that there are ultraparticles which cannot be created by physical operations at finite scales, into a precise definition. To this end we consider the scaling limit $\mathcal{F}_{0}$ of the algebra $\mathcal{F}$ of charged physical fields. It can be computed by the same methods as outlined above for the observables. The algebra $\mathcal{F}_{0}$ is an extension of the algebra $\mathcal{A}_{0}$ contained in $\mathcal{F}^{0}, \mathcal{F}_{0} \subset \mathcal{F}^{0}$, and this inclusion may be proper. We say:

An ultraparticle is confined if all of its state vectors are orthogonal to the set of vectors $\mathcal{F}_{0}\left|V_{0}\right\rangle$.

Indeed, such an ultraparticle cannot be created by the underlying fields in $\mathcal{F}$ at the relevant scale and therefore does not appear in the "physical spectrum." An interesting special case occurs if the elements of $\mathcal{F}_{0}$ are pointwise invariant under some subgroup $C_{0} \subset G_{0}$. Then we have:

Any ultraparticle carrying a non-trivial charge with regard to $C_{0}$ is confined.

Note that our definition of confinement of ultraparticles does not refer to any dynamical properties. If $\mathcal{F}$ is generated by local operators (what we assume here) the condition can be tested in arbitrarily small spacetime regions.

We have reached now a point where we can try to answer the question raised in the title. To this end let us recall some basic facts of hadronic physics. The spectrum of hadrons, the scaling behaviour of collision cross sections, the jet structure of final states in collision processes etc. seem to indicate that the structure of hadronic matter at small scales can be understood in terms of particle-like entities. Without alluding to any particular model, let us call them quarks and gluons.

On the theoretical side these experimental facts seem to call for a description in terms of a set $\Sigma_{0}$ of ultraparticles. So the theory one is looking for has to be a free theory at small scales. Taking also into account the charges and statistics of hadrons, decay rates of mesons, ratios of total cross sections etc., one is led to the idea that the group of ultrasymmetries $G_{0}$ of the theory contains a "flavour group" $F_{0}$ and a "colour group" $C_{0}$. In particular, quarks and gluons carry nontrivial charges with regard to $C_{0}$. On the other hand, states carrying a colour charge apparently cannot be created by physical operations. So quarks and gluons ought to be confined in the desired theory.

Thus a reasonable theory of hadronic physics should have the following general structure in the scaling limit: the algebra $\mathcal{F}^{0}$ should be generated by $G_{0^{-}}$ multiplets of free massless Dirac and electromagnetic fields, describing the ultraparticles quarks and gluons. In order that these ultraparticles are confined, the scaling limit $\mathcal{F}_{0}$ of the algebra of charged physical fields $\mathcal{F}$ should be pointwise 
invariant under the action of $C_{0}$.

The latter condition imposes a non-trivial dynamical constraint on the theory in demand, but there are good reasons to believe that a solution to this problem is already known: one regards the fields generating $\mathcal{F}^{0}$ as the kinematical field content of the underlying theory and "gauges" the colour group $C_{0}$ by introducing corresponding (self)couplings between the electromagnetic and Dirac fields in $\mathcal{F}^{0}$. The result is of course quantum chromodynamics. It is an asymptotically free theory and one may therefore hope that one can recover from the observables in the scaling limit the structure which one has taken as a basis in its heuristic derivation. Admittedly, this is fiction at present since the theory has not yet been rigorously constructed. But as all one needs to know are the short distance properties of the theory it suffices to construct it in a finite volume, no matter how small. Since there is progress in this respect [22] it may well be possible to decide on this question in the foreseeable future.

If quantum chromodynamics turns out to have an ultraparticle and ultrasymmetry structure as outlined above, then quarks, gluons and the colour symmetry are described by the theory as physical facts and the same holds true for the notion of confinement. As these features can entirely be recovered from the observables they cannot be "gauged away", not even by duality transformations.

The theoretical interpretation of these structures in terms of gauge fields may be ambiguous, however. As the example of the Schwinger model, discussed in the subsequent section, and the more recently discovered gauge theories show, notions such as "strong colour-electric forces" can be theoretical fictions whithout any intrinsic meaning. One can change the theoretical explanations of the observable features of these models in a radical, almost contradictory way. On the other hand, quarks, gluons and the colour group seem to be elements of physical reality which admit, on the theoretical side, an unambiguous description in terms of ultraparticles and ultrasymmetries.

\section{The Schwinger model at small scales}

We have presented our approach to the short distance analysis of quantum field theories in a general setting in order to make clear that the method has the character of a universal algorithm. In this section we apply this method to the simplest model which seems to cause some problems as far as its physical interpretation is concerned, the Schwinger model. What is the ultraparticle and ultrasymmetry content of this theory? The answer may seem obvious, but there are a few surprises.

As has been mentioned in the Introduction, the algebra $\mathcal{A}$ of local observables in the Schwinger model is generated by the free scalar field $\phi$ in two spacetime dimensions with mass $m>0$ [3]. Actually, the algebra $\mathcal{A}$ has also a center. But we may disregard this center here since it remains unaffected under scaling transformations. More concretely, we pick any one of the pure vacuum states of the Schwinger model and restrict the observables to the corresponding vacuum 
subspace. The elements of the center then become multiples of the identity and the vacuum can be identified with the vacuum vector $|V\rangle$ of the free field $\phi$.

We first note that there are no charged states in the model, that is states satisfying the selection criteria described in Sec.2, which do not lie in the vacuum Hilbert space. This fact can be extracted for example from the results in [23]. So the global symmetry group $G$ in the Schwinger model is trivial and there holds $\mathcal{F}=\mathcal{A}$.

Next, let us turn to the computation of the scaling limit of the observables. As all observables are functions of $\phi$, it suffices to consider the operators (cf. relation $(2.4))$

$$
A_{\lambda} \doteq \int d^{2} y g(y) \exp \left(i \int d^{2} x f(x-y) N_{\lambda} \phi(\lambda x)\right), \quad \lambda>0
$$

where $f, g$ are arbitrary real test functions and $N_{\lambda}$ is an arbitrary positive normalization factor. We have to compute the corresponding expectation values $\left\langle V\left|A_{\lambda}^{(1)} A_{\lambda}^{(2)} \cdots A_{\lambda}^{(n)}\right| V\right\rangle$, where $A_{\lambda}^{(j)}$ has the same form as $A_{\lambda}$ with $f, g, N_{\lambda}$ replaced by arbitrary test functions and normalization factors $f^{(j)}, g^{(j)}, N_{\lambda}^{(j)}$, and to proceed to the limit $\lambda \rightarrow 0$.

In view of the $c$-number commutation relations of the free field $\phi$ there holds

$$
\begin{aligned}
A_{\lambda}^{(1)} A_{\lambda}^{(2)} \cdots A_{\lambda}^{(n)}= & \int \cdots \int \prod_{j=1}^{n} d^{2} y_{j} g^{(j)}\left(y_{j}\right) \eta_{\lambda}\left(y_{1}, \ldots y_{n}\right) \times \\
& \times \exp \left(i \int d^{2} x\left(\sum_{k=1}^{n} N_{\lambda}^{(k)} f^{(k)}\left(x-y_{k}\right)\right) \phi(\lambda x)\right),
\end{aligned}
$$

where $\eta_{\lambda}\left(y_{1}, \ldots y_{n}\right)$ is a phase factor given by

$$
\begin{aligned}
& \eta_{\lambda}\left(y_{1}, \ldots y_{n}\right)= \\
= & \exp \left(2 \pi i \operatorname{Im} \sum_{k>l} N_{\lambda}^{(k)} N_{\lambda}^{(l)} \int \frac{d \boldsymbol{p}}{2 \sqrt{\boldsymbol{p}^{2}+\lambda^{2} m^{2}}} \widetilde{\widetilde{f^{(k)}}(p(\lambda))} \widetilde{f^{(l)}}(p(\lambda)) e^{i p(\lambda)\left(y_{l}-y_{k}\right)}\right) .
\end{aligned}
$$

Here $\tilde{f}$ denotes the Fourier transform of $f$ and $p(\lambda) \doteq\left(\sqrt{\boldsymbol{p}^{2}+\lambda^{2} m^{2}}, \boldsymbol{p}\right)$. Thus the vacuum expectation value of the operator (4.2) is given by

$$
\begin{aligned}
& \left\langle V\left|A_{\lambda}^{(1)} A_{\lambda}^{(2)} \cdots A_{\lambda}^{(n)}\right| V\right\rangle=\int \cdots \int \prod_{j=1}^{n} d^{2} y_{j} g^{(j)}\left(y_{j}\right) \eta_{\lambda}\left(y_{1}, \ldots y_{n}\right) \times \\
& \quad \times \exp \left(-\pi \int \frac{d \boldsymbol{p}}{2 \sqrt{\boldsymbol{p}^{2}+\lambda^{2} m^{2}}} \mid \sum_{k=1}^{n} N_{\lambda}^{(k)} e^{\left.\left.i p(\lambda) y_{k} \widetilde{f^{(k)}}(p(\lambda))\right|^{2}\right) .}\right.
\end{aligned}
$$

Let us analyze the possible behaviour of this expression for $\lambda \rightarrow 0$.

(a) If there is some $N_{\lambda}^{(k)}$ which diverges as $\lambda \rightarrow 0$ the expression vanishes, no matter how the other normalization factors behave in this limit. This is a consequence of the dominated convergence theorem, applied to the $y$-integrations, and the fact that the exponential function in (4.4) vanishes for almost any choice of $y_{1}, \ldots y_{n}$. 
(b) Next let us assume that all $N_{\lambda}^{(k)}$ converge as $\lambda \rightarrow 0$. Then $\eta_{\lambda}\left(y_{1}, \ldots y_{n}\right)$ converges, too. Note that the logarithmic divergence appearing in (4.3) in the integration if $\lambda=0$ does not cause any problems since the divergent contribution is real. The properties of the exponential function in (4.4) are more subtle, however. First, it is obvious that the logarithmic divergence of the integral now becomes effective if $\sum_{k=1}^{n} N_{0}^{(k)} \widetilde{f^{(k)}}(0) \neq 0$. Then the expression (4.4) vanishes in the limit $\lambda \rightarrow 0$. If the sum is equal to 0 , one finds by an asymptotic expansion that the exponential function in (4.4) can be replaced in the limit $\lambda \rightarrow 0$ by

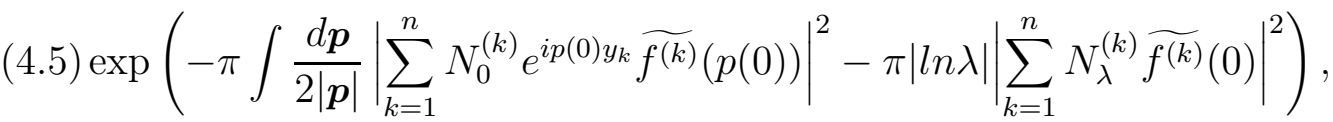

where $p(0)=(|\boldsymbol{p}|, \boldsymbol{p})$. Because of the appearance of the divergent factor $|\ln \lambda|$ it is apparent that also the rate of convergence of the normalization factors $N_{\lambda}^{(k)}$ matters. This fact leaves room for some interesting phenomenon, as we shall see.

If one replaces in (4.4) the exponential function by (4.5) and $\eta_{\lambda}\left(y_{1}, \ldots y_{n}\right)$ by its limit $\eta_{0}\left(y_{1}, \ldots y_{n}\right)$ one finds that the resulting expression has a very simple interpretation. To exhibit this fact, let

$$
\hat{A}_{0}^{(k)} \doteq \int d^{2} y g^{(k)}(y) \exp \left(i \int d^{2} x f^{(k)}(x-y) N_{0}^{(k)} \phi_{0}(x)\right)
$$

where $\phi_{0}$ is the free massless scalar field, and let $\left|\hat{V}_{0}\right\rangle$ be the corresponding vacuum vector. We recall that the exponential functions of the smoothed-out field $\phi_{0}$ (Weyl-operators) can be defined on a vacuum Hilbert space with positive metric, cf. also the remarks below. Next, let $C$ be some classical random variable with spectrum $\mathbb{R}$ and let $\langle\cdot\rangle_{G}$ be the Gaussian state (probability measure) given by

$$
\langle\exp (i \zeta C)\rangle_{G} \doteq e^{-\zeta^{2} / 2}, \quad \zeta \in \mathbb{R} .
$$

We set

$$
\check{A}_{\lambda}^{(k)} \doteq \exp \left(i|2 \pi \ln \lambda|^{1 / 2}\left(N_{\lambda}^{(k)}-N_{0}^{(k)}\right) \widetilde{f^{(k)}}(0) C\right), \quad \lambda>0 .
$$

It then follows from the preceding computations that, apart from terms which vanish for $\lambda \rightarrow 0$, there holds

$$
\left\langle V\left|A_{\lambda}^{(1)} \cdots A_{\lambda}^{(n)}\right| V\right\rangle=\left\langle\hat{V}_{0}\left|\hat{A}_{0}^{(1)} \cdots \hat{A}_{0}^{(n)}\right| \hat{V}_{0}\right\rangle\left\langle\check{A}_{\lambda}^{(1)} \cdots \check{A}_{\lambda}^{(n)}\right\rangle_{G}
$$

if $\sum_{k=1}^{n} N_{0}^{(k)} \widetilde{f^{(k)}}(0)=0$. If this sum is different from zero the right hand side of (4.9) has to be replaced by 0 . Thus we see that at small scales the theory can be interpreted in terms of a free massless scalar field and an independent classical random variable $C$. So there appears a new degree of freedom at small scales.

(c) Let us finally discuss the case where the normalization factors $N_{\lambda}^{(k)}$ have a behaviour at $\lambda=0$ such that the expectation values (4.4) do not converge. Then there still holds

$$
\left|\left\langle V\left|A_{\lambda}^{(1)} \cdots A_{\lambda}^{(n)}\right| V\right\rangle\right| \leq \prod_{k=1}^{n} \int d^{2} y\left|g^{(k)}(y)\right|
$$


so the expectation values are uniformly bounded for any choice of the normalization factors. It thus follows from elementary calculus (Heine-Borel theorem) that there always exist subsequences $\lambda_{i} \rightarrow 0$ such that the expectation values converge. One is then in the framework either of case (a) or of case (b), so one gets nothing new. The non-convergence of the expectation values simply means that one has picked at different scales observables which test rather different properties of the state. But by proceeding to subsequences one can always correct this inappropriate choice.

These results illustrate the general method of the scaling algebra [8]. It is an algorithm which does not require any a priori information about the "correct" normalization factor $Z_{\lambda}$. As a matter of fact, if we had plugged into relation (4.1) the - according to the canonical dimension of the field $\phi$-obvious choice $N_{\lambda}=1$ we would have missed the interesting point that the field contains also a classical contribution $C$ with anomalous dimension. By our unbiased approach this fact has come to light.

Summing up, we see that the algebra of observables $\mathcal{A}$ in the Schwinger model has in the scaling limit the form of a tensor product, $\mathcal{A}_{0}=\hat{\mathcal{A}}_{0} \otimes \check{\mathcal{A}}_{0}$, where $\hat{\mathcal{A}}_{0}$ is the algebra generated by the free massless scalar field $\phi_{0}$ in Weyl form and $\check{\mathcal{A}}_{0}$ is an abelian algebra generated by the classical variable $C$. (Note that the center $1 \otimes \check{\mathcal{A}}_{0}$ of $\mathcal{A}_{0}$ has to be distinguished from the center of the original algebra of observables, which is still present as an additional "dummy variable".) Moreover, the pure vacuum state on $\mathcal{A}$ becomes a mixed state on $\mathcal{A}_{0}$ which may be interpreted as a vacuum state containing a condensate of zero energy Bosons.

We mention as an aside that the latter phenomenon is a peculiarity of the two-dimensional world. In higher dimensions the scaling limit of a pure vacuum state is always pure [8].

Let us turn now to the determination of the ultraparticles and ultrasymmetries in the Schwinger model. We do not aim here at a complete description of $G_{0}, \Sigma_{0}$ and restrict ourselves to showing that there appear charged physical states in the scaling limit. To some extent we can follow here ideas already expounded in the literature, cf. for example [24].

The center of $\mathcal{A}_{0}$ is of little interest, in particular since its appearance is a peculiarity of two dimensional theories. So we need not think up new states on this part of the algebra and may restrict our attention to states $\left\langle E_{0}|\cdot| E_{0}\right\rangle$ on $\mathcal{A}_{0}$ of the form

$$
\left\langle E_{0}\left|A_{0}^{(1)} \cdots A_{0}^{(n)}\right| E_{0}\right\rangle=\left\langle\hat{E}_{0}\left|\hat{A}_{0}^{(1)} \cdots \hat{A}_{0}^{(n)}\right| \hat{E}_{0}\right\rangle\left\langle\check{A}_{0}^{(1)} \cdots \check{A}_{0}^{(n)}\right\rangle_{G}
$$

where $\left\langle\hat{E}_{0}|\cdot| \hat{E}_{0}\right\rangle$ is a suitable functional on the algebra $\hat{\mathcal{A}}_{0}$ generated by the exponential functions of the smoothed-out free massless field $\phi_{0}$. In view of the fact that $\langle\cdot\rangle_{G}$ is kept fixed we may ignore in the following the center $1 \otimes \check{\mathcal{A}}_{0}$ and identify the algebra $\mathcal{A}_{0}$ with its free field part $\hat{\mathcal{A}}_{0}$.

The specification of functionals $\left\langle\hat{E}_{0}|\cdot| \hat{E}_{0}\right\rangle$ on $\hat{\mathcal{A}}_{0}$ is greatly simplified by the fact that the algebraic relation (4.2) holds also for the operators $\hat{A}_{0}^{(k)}$ if one replaces $N_{\lambda}^{(k)}, \eta_{\lambda}\left(y_{1}, \ldots y_{n}\right), \phi(\lambda x)$ by $N_{0}^{(k)}, \eta_{0}\left(y_{1}, \ldots y_{n}\right)$ and $\phi_{0}(x)$, respectively. It 
therefore suffices to specify the functionals for a single Weyl-operator of the form $\hat{W}_{0}(f)=\exp \left(i \int d^{2} x f(x) \phi_{0}(x)\right)$, where $f$ is an arbitrary real test function.

The vacuum state on $\hat{\mathcal{A}}_{0}$ is fixed by setting

$$
\left\langle\hat{V}_{0}\left|\hat{W}_{0}(f)\right| \hat{V}_{0}\right\rangle=\left\{\begin{array}{ccc}
\exp \left(-\pi \int \frac{d \boldsymbol{p}}{2|\boldsymbol{p}|}|\tilde{f}(p(0))|^{2}\right) & \text { if } & \tilde{f}(0)=0 \\
0 & \text { if } & \tilde{f}(0) \neq 0
\end{array}\right.
$$

where $p(0)=(|\boldsymbol{p}|, \boldsymbol{p})$ as before. ${ }^{5}$ The simplest class of states on $\hat{\mathcal{A}}_{0}$ next to the vacuum are the so-called coherent states. They give rise to expectation values of the form

$$
\left\langle\hat{E}_{0}\left|\hat{W}_{0}(f)\right| \hat{E}_{0}\right\rangle=e^{i L(f)}\left\langle\hat{V}_{0}\left|\hat{W}_{0}(f)\right| \hat{V}_{0}\right\rangle
$$

where $L$ is a linear functional on the space of real test functions which vanishes on all functions of the form $f(x)=\square g(x)$. Phrased differently, $L$ is a weak solution of the wave equation. Picking any such $L$ one obtains from (4.13) with the help of relation (4.2) a state on $\hat{\mathcal{A}}_{0}$ which, by the reconstruction theorem, gives rise to a representation of $\hat{\mathcal{A}}_{0}$ on some Hilbert space. The problem is that this representation may not describe elementary systems in the sense of the selection criterion given in Sec.2. So one has to determine the subset of functionals $L$ which are admissible in that sense.

Instead of giving the complete solution of this problem it may suffice here to exhibit an interesting class of functionals $L$ which comply with the selection criterion. Let $\rho(\boldsymbol{x}), \boldsymbol{x} \in \mathbb{R}$, be any real, symmetric test function with support in the interval $[-r, r]$, say, and let $\int d \boldsymbol{x} \rho(\boldsymbol{x})=q$, where $q \neq 0$ is some fixed "charge". We put

$$
L(f) \doteq \sqrt{2 \pi} \int d \boldsymbol{p} \frac{\tilde{\rho}(\boldsymbol{p})}{2 i \boldsymbol{p}}(\tilde{f}(p(0))-\tilde{f}(-p(0)))
$$

This functional is well-defined, real and linear and there holds $L(\square g)=0$.

One can show that the representation of $\hat{\mathcal{A}}_{0}$ which is induced by the state (4.13) for the given functional $L$ admits energy-momentum operators which are the generators of space-time translations and satisfy the relativistic spectrum condition. Since we are dealing with coherent states this fact is almost obvious. For example, the expectation value of the energy operator in the state fixed by $L$ is given by $(1 / 2) \int d \boldsymbol{x}|\rho(\boldsymbol{x})|^{2}$. A rigorous proof of the assertion is obtained by applying standard methods, cf. for example [25].

Next we have to determine the configuration space properties of the functional $L$. To this end we introduce the function

$$
\tilde{h}(\boldsymbol{p}) \doteq \frac{1}{2}(\tilde{f}(|\boldsymbol{p}|, \boldsymbol{p})+\tilde{f}(-|\boldsymbol{p}|, \boldsymbol{p}))
$$

\footnotetext{
${ }^{5}$ In view of the discontinuity at $f=0$ this vacuum state is a so-called non-regular state. As a consequence, the smoothed-out field operators $\phi_{0}(f)$ cannot be defined on the corresponding Hilbert space if $\tilde{f}(0) \neq 0$, in contrast to the Weyl-operators.
} 
and note that the functional $L$ can be represented in the form

$$
L(f)=-\sqrt{\frac{\pi}{2}} \int d \boldsymbol{x}(h(\boldsymbol{x})-h(-\boldsymbol{x})) \int_{-\infty}^{\boldsymbol{x}} d \boldsymbol{y} \rho(\boldsymbol{y})
$$

since $\rho$ is symmetric. Now let $a=\left(a_{0}, \boldsymbol{a}\right) \in \mathbb{R}^{2}, a_{0}>0$, and let $f$ be any test function which has support in the region $\left\{x=\left(x_{0}, \boldsymbol{x}\right) \in \mathbb{R}^{2}:\left|x_{0}\right|+|\boldsymbol{x}-\boldsymbol{a}|<a_{0}\right\}$. Then the Fourier transform $h$ of $\tilde{h}$ has support in the interval $\left[\boldsymbol{a}-a_{0}, \boldsymbol{a}+a_{0}\right]$. Hence if this interval does not intersect with $[-r, r]$, we infer from $(4.16)$, bearing in mind that $\int d \boldsymbol{x} \rho(\boldsymbol{x})=q$, that

$$
L(f)=\mp \pi q \tilde{f}(0) \quad \text { if } \quad a_{0}< \pm(\boldsymbol{a}-r) .
$$

This result can be rephrased in more geometrical terms. If one regards the interval $[-r, r]$ as a region in Minkowski space $\mathbb{R}^{2}$ at time $x_{0}=0$, then relation (4.17) holds if $f$ has support in the right, respectively left spacelike complement of that region.

This fact has interesting consequences for the localization properties of the corresponding state. Plugging the result (4.17) into relation (4.13) we find that

$$
\left\langle\hat{E}_{0}\left|\hat{W}_{0}(f)\right| \hat{E}_{0}\right\rangle=e^{\mp i \pi q \tilde{f}(0)}\left\langle\hat{V}_{0}\left|\hat{W}_{0}(f)\right| \hat{V}_{0}\right\rangle .
$$

If $\tilde{f}(0) \neq 0$, then $\left\langle\hat{V}_{0}\left|\hat{W}_{0}(f)\right| \hat{V}_{0}\right\rangle=0$, hence there holds also $\left\langle\hat{E}_{0}\left|\hat{W}_{0}(f)\right| \hat{E}_{0}\right\rangle=0$. On the other hand, if $\tilde{f}(0)=0$ it follows from (4.18) that $\left\langle\hat{E}_{0}\left|\hat{W}_{0}(f)\right| \hat{E}_{0}\right\rangle=$ $\left\langle\hat{V}_{0}\left|\hat{W}_{0}(f)\right| \hat{V}_{0}\right\rangle$. Hence the two states $\left\langle\hat{E}_{0}|\cdot| \hat{E}_{0}\right\rangle$ and $\left\langle\hat{V}_{0}|\cdot| \hat{V}_{0}\right\rangle$ coincide on all operators $\hat{W}_{0}(f)$, provided $f$ has support in the right or left spacelike complement of the interval $[-r, r]$ at time $x_{0}=0$. In this sense the state $\left\langle\hat{E}_{0}|\cdot| \hat{E}_{0}\right\rangle$ corresponds to an elementary excitation of the vacuum.

The conclusion that this excitation is strictly localized in the region $[-r, r]$ would be wrong, however. This can be seen if one chooses a test function $f$ of the form $f=f_{+}+f_{-}$, where $f_{ \pm}$have support in the right and left spacelike complement of the interval $[-r, r]$ at time $x_{0}=0$, respectively. Because of the linearity of the functional $L$ one obtains in this case

$$
\left\langle\hat{E}_{0}\left|\hat{W}_{0}(f)\right| \hat{E}_{0}\right\rangle=e^{-i \pi q\left(\widetilde{f_{+}}(0)-\widetilde{f_{-}}(0)\right)}\left\langle\hat{V}_{0}\left|\hat{W}_{0}(f)\right| \hat{V}_{0}\right\rangle .
$$

Picking $f$ with $\widetilde{f_{+}}(0)+\widetilde{f_{-}}(0)=0$ and $\widetilde{f_{ \pm}}(0) \neq 0$ it is thus possible to discriminate the two functionals in the spacelike complement of any bounded region, no matter how large.

It remains to be shown that the state $\left\langle\hat{E}_{0}|\cdot| \hat{E}_{0}\right\rangle$ cannot be represented by a vector in the Hilbert space of neutral states. This follows by standard arguments, using the fact that the function $|\tilde{\rho}(\boldsymbol{p})|^{2} /|\boldsymbol{p}|$ is not integrable at $\boldsymbol{p}=0$. Instead of giving a formal proof the following argument may be more instructive. Consider the identically conserved "electric" current $j_{\mu}(x)=\partial^{\nu} \varepsilon_{\mu \nu} \phi_{0}(x)$, where $\partial^{\nu}$ denotes the space-time derivatives and $\varepsilon_{\mu \nu}$ the antisymmetric tensor. By a straightforward computation one finds that

$$
\left\langle\hat{E}_{0}\left|j_{0}(x)\right| \hat{E}_{0}\right\rangle=\frac{1}{\sqrt{2 \pi}} \int d \boldsymbol{p} \tilde{\rho}(\boldsymbol{p}) \cos \left(|\boldsymbol{p}| x_{0}\right) \cos (\boldsymbol{p} \boldsymbol{x})
$$


and consequently

$$
\int_{x_{0}=\text { const }} d \boldsymbol{x}\left\langle\hat{E}_{0}\left|j_{0}(x)\right| \hat{E}_{0}\right\rangle=q .
$$

So the functional $\left\langle\hat{E}_{0}|\cdot| \hat{E}_{0}\right\rangle$ describes a charged state. On the other hand, since "Gauss' law" $j_{0}=\partial_{1} \phi_{0}$ holds for the current $j_{\mu}(x)$, all the locally generated vectors in the space $\hat{\mathcal{A}}_{0}\left|\hat{V}_{0}\right\rangle$ carry zero charge.

Summing up, we have shown that the algebra $\hat{\mathcal{A}}_{0}$ admits states describing charged elementary systems. Intuitively speaking, these systems carry a welllocalized "electric" charge to which an infinite string is attached which is strongly fluctuating. One cannot decide by measurements with certainty whether this string emanates to plus or minus spacelike infinity. The states look in the right and left spacelike complement of the localization region of the charge like the vacuum, but the presence of the string can be established if one makes suitable coincidence measurements in both regions, cf. relation (4.19). Thus these states describe the perhaps simplest example of a quantum topological charge in the sense of $[12]$.

Let us turn now to the determination of the charged fields $\mathcal{F}^{0}$, the ultrasymmetries $G_{0}$ and the set $\Sigma_{0}$ of ultraparticles, which are fixed by the class of states which we have constructed. Here we cannot rely on the general results of Doplicher and Roberts which hold only in physical spacetime $\mathbb{R}^{4}$. But the necessary computations are simple enough to be carried out explicitly.

The charged fields in $\mathcal{F}^{0}$ creating the vectors $\left|\hat{E}_{0}\right\rangle$ from the vacuum $\left|\hat{V}_{0}\right\rangle$ are obtained by a limiting procedure from operators in $\hat{\mathcal{A}}_{0}$ (akin to bosonization formulas). They can formally be represented according to

$$
\hat{\psi}_{\rho}(x) \doteq \lim _{R \rightarrow \infty} \exp \left(i \phi_{0}\left(\rho_{R, x}\right)\right), \quad x \in \mathbb{R}^{2},
$$

where the Fourier transform of $\rho_{R, x}$ is given by

$$
\widetilde{\rho_{R, x}}(p)=\frac{1}{\sqrt{2 \pi}} p_{0} \tilde{\rho}(\boldsymbol{p}) \frac{1}{\boldsymbol{p}}\left(1-\frac{\sin (\boldsymbol{p} R)}{\boldsymbol{p} R}\right) e^{i p x} .
$$

Note that $\rho_{R, x}$ is real and has compact support. The rule of the game is that one first has to compute the expectation values involving these operators for fixed $R$ and then to proceed to the limit $R \rightarrow \infty$. This procedure corresponds to the process of "shifting a compensating charge behind the moon", mentioned in Sec.2.

By a calculation analogous to the case of relation (4.4) one finds that the expectation value of any product $\hat{\psi}_{\rho_{1}}\left(x_{1}\right) \cdots \hat{\psi}_{\rho_{n}}\left(x_{n}\right)$ of field operators in the vacuum state $\left\langle\hat{V}_{0}|\cdot| \hat{V}_{0}\right\rangle$ vanishes if $q_{1}+\cdots+q_{n} \neq 0$. Hence the mappings

$$
\hat{\psi}_{\rho}(x) \rightarrow e^{i \xi q} \hat{\psi}_{\rho}(x), \quad \xi \in \mathbb{R}
$$

define global gauge transformations of the fields in $\mathcal{F}^{0}$. Moreover, the observables in $\mathcal{A}_{0}$ are invariant under these transformations. Thus, according to our terminology, the gauge group generated by the transformations (4.24) is an ultrasymmetry of the Schwinger model. 
It remains to determine the ultraparticle content of the model. To this end let us first have a look at the energy-momentum spectrum of the vectors $\left|\hat{E}_{0}\right\rangle=$ $\hat{\psi}_{\rho}\left|\hat{V}_{0}\right\rangle$ which can be extracted from the two-point function

$$
\left\langle\hat{V}_{0}\left|\hat{\psi}_{\rho}^{*} \hat{\psi}_{\rho}(x)\right| \hat{V}_{0}\right\rangle=\exp \left(-\int \frac{d \boldsymbol{p}}{2|\boldsymbol{p}|}|\tilde{\rho}(\boldsymbol{p})|^{2}\left(1-e^{i p(0) x}\right)\right)
$$

One can show that the Fourier transform of this function does not have a discrete ( $\delta$-function) mass-shell contribution, the reason being again the non-integrability of the function $|\tilde{\rho}(\boldsymbol{p})|^{2} /|\boldsymbol{p}|$ at $\boldsymbol{p}=0$. Thus, according to the conventional definition of particle states, the charged states do not seem to have a particle interpretation. But this conclusion would be premature. In order to see this let us look more closely at the $x$-dependence of the expectation value (4.20) which can easily be computed. It is given by

$$
\left\langle\hat{E}_{0}\left|j_{0}(x)\right| \hat{E}_{0}\right\rangle=\frac{1}{2}\left(\rho\left(\boldsymbol{x}+x_{0}\right)+\rho\left(\boldsymbol{x}-x_{0}\right)\right) .
$$

Hence the charge density of the state $\left|\hat{E}_{0}\right\rangle$ propagates with the velocity of light through spacetime and is localized in regions of fixed size. It is therefore meaningful to say that the state described by $\left|\hat{E}_{0}\right\rangle$ contains a charged massless ultraparticle. The absence of a discrete contribution in the mass spectrum of (4.25) is due to the fact that this particle cannot be separated from clouds of neutral particles accompanying it. In view of the fact that "Gauss' law" holds for the charge this is not a surprise [14].

Thus the charged ultraparticles in the Schwinger model turn out to be infraparticles in the sense of [26] as well. This observation shows that the conventional (Wigner) particle concept may not always be sufficient to describe the ultraparticles in a theory. Yet since there exists a systematic way to uncover all particle like structures from the observables, including infraparticles, cf. [27] and [7], this point does not provide an obstacle to our general approach.

The second ultraparticle appearing in the Schwinger model is neutral and massless. Its states can be generated from the vacuum by the observable $\partial_{\mu} \phi_{0}(x)$. In contrast to the charged ultraparticle whose state vectors are orthogonal to all vectors in $\mathcal{F}_{0}\left|\hat{V}_{0}\right\rangle=\mathcal{A}_{0}\left|\hat{V}_{0}\right\rangle$, this particle is not confined. It is what remains of the massive particle in the scaling limit.

This completes our analysis of the observables of the Schwinger model in the scaling limit, cf. [28] for a more detailed account. The results nicely illustrate the general picture of ultraparticles, outlined in Sec.3. They show that "electrically" charged ultraparticles appear in the model at small scales which have no counterpart at large scales, so they are confined. Within the theoretical setting this must be regarded as an observable fact.

What seems to be somewhat arbitrary, however, is the theoretical explanation of this phenomenon, offered by the Schwinger model. It relies on the picture that the electric force is strong at large distances and binds opposite charges into massive bound states. Since the theory is asymptotically free one may then 
argue that the force is turned off at small scales so that charged ultraparticles can appear as physical states in the scaling limit.

This is an illustrative and consistent explanation of the observable features of the Schwinger model, but it does not have any more predictive power than the explanation suggested by massive free field theory. As there are no forces present in the latter model, that explanation looks rather different, however.

From the point of view of free field theory the appearance of charged elementary systems in the scaling limit is a pure quantum effect. In order to exhibit this mechanism let us proceed from the field $\phi_{\lambda}$ at scale $\lambda$ to the corresponding time $x_{0}=0$ field $\varphi_{\lambda}$ and its canonically conjugate field (time derivative) $\pi_{\lambda}$. If one integrates $\pi_{\lambda}$ with a real test function $g$ one finds that for any normalized state vector $|S\rangle$ in the underlying Fock space there holds [29]

$$
\left\langle S\left|\pi_{\lambda}(g)^{2}\right| S\right\rangle \leq \int d \boldsymbol{p} \sqrt{\boldsymbol{p}^{2}+\lambda^{2} m^{2}}|\tilde{g}(\boldsymbol{p})|^{2} \cdot\langle S|(2 N+1)| S\rangle,
$$

where $N$ is the particle number operator. In view of the canonical commutation relations $\left[\varphi_{\lambda}(f), \pi_{\lambda}(g)\right]=i \int d \boldsymbol{p} \tilde{f}(-\boldsymbol{p}) \tilde{g}(\boldsymbol{p}) \cdot 1$ one obtains from this estimate according to the uncertainty principle the inequality

$$
\begin{aligned}
\left\langle S\left|\varphi_{\lambda}(f)^{2}\right| S\right\rangle & -\left\langle S\left|\varphi_{\lambda}(f)\right| S\right\rangle^{2} \geq \\
& \geq \sup _{g} \frac{\left|\int d \boldsymbol{p} \tilde{f}(-\boldsymbol{p}) \tilde{g}(\boldsymbol{p})\right|^{2}}{4 \int d \boldsymbol{p} \sqrt{\boldsymbol{p}^{2}+\lambda^{2} m^{2}}|\tilde{g}(\boldsymbol{p})|^{2} \cdot\langle S|(2 N+1)| S\rangle} \\
& =\frac{1}{4} \int \frac{d \boldsymbol{p}}{\sqrt{\boldsymbol{p}^{2}+\lambda^{2} m^{2}}}|\tilde{f}(\boldsymbol{p})|^{2} \cdot \frac{1}{\langle S|(2 N+1)| S\rangle} .
\end{aligned}
$$

Hence if $\tilde{f}(0) \neq 0$, the fluctuations of $\varphi_{\lambda}(f)$ become infinitely large in the scaling limit, which makes it impossible to discriminate different states by these operators (the corresponding measurements would have infinite error bars).

As a result, certain field configurations which can be distinguished at finite scales become indistinguishable in the scaling limit. If one adds for example to some state $\langle S|\cdot| S\rangle$ an external field $F$ at time $x_{0}=0$ this amounts to replacing in the original expectation values the operator $\varphi_{\lambda}(f)$ by $\varphi_{\lambda}(f)+F(f) \cdot 1$. It therefore does not affect the fluctuations of $\varphi_{\lambda}(f)$. Consequently, the value of $F(f)$ becomes meaningless at small scales if $\tilde{f}(0) \neq 0$. On the other hand, for test functions $f$ with $\tilde{f}(0)=0$ only the spatial derivative $\partial_{1} F$ of the field $F$ is tested. So fields $F$ differing by a constant cannot be distinguished at small scales and kink-like field configurations, which contain an infinite amount of energy in their asymptotically constant pieces, look in the scaling limit like localized excitations with finite energy. Thus quantum effects provide an alternative explanation of the appearance of ultraparticles and ultrasymmetries in the Schwinger model and the gauge fields may then be viewed as nothing more but some choice of integration variables in a functional integral. 


\section{Summary}

We have presented in this article a general method which allows one to extract from the local observables in a given theory the structure and interpretation of the theory at very small spatio-temporal scales. The necessary steps are indicated in the following diagram:

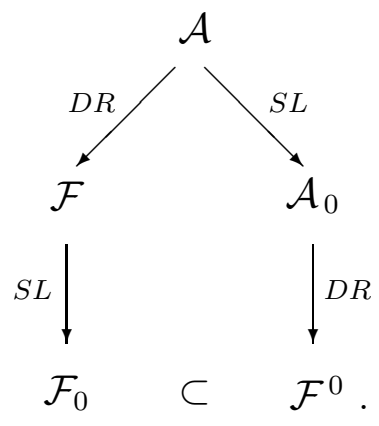

Here DR stands for the Doplicher-Roberts construction of charged physical fields from local observables [9] and SL denotes the novel method of computing scaling limits, invented in [8]. As is indicated by the diagram, the whole structure is completely fixed once the algebra $\mathcal{A}$ of observables is given.

The ultraparticles and ultrasymmetries appearing in the scaling limit can be extracted from the algebra $\mathcal{F}^{0}$ and are thus an intrinsic property of the underlying theory which must be regarded as an (in principle) observable fact. Moreover, one can distinguish between confined and non-confined ultraparticles by comparing the algebras $\mathcal{F}_{0}$ and $\mathcal{F}^{0}$. Loosely speaking, there is room for the phenomenon of confinement of ultraparticles in the theoretical setting since the operations DR and SL do not need to commute.

These results do not rely in any way on the existence of gauge fields. As a matter of fact, such an extension of an algebra of observables $\mathcal{A}$ to a theoretical superstructure may be ambiguous, as one knows from various examples.

The general picture of the short distance structure of asymptotically free, confining theories which emerges from these results has been illustrated by the Schwinger model. It seems feasible that with some effort it can also be established in physically more interesting theories, such as quantum chromodynamics. 


\section{Acknowledgements}

I enjoyed discussions with J. Gomis, H. Joos and I. Ojima on the subject of this paper and I am very grateful to the members of RIMS and its director H. Araki for their hospitality and care during my visit to Kyoto University. I also acknowledge a research grant from the Japan Society for the Promotion of Science (JSPS) and financial support from the Deutsche Forschungsgemeinschaft (DFG).

\section{References}

[1] N. Seiberg, The power of duality - Exact results in $4 D$ SUSY field theory. Preprint hep-th/950607n, to appear in the Proceedings of PASCOS 95.

[2] R. Haag, Local quantum physics, Springer, Berlin, 1992.

[3] J.H. Lowenstein, J.A. Swieca, Quantum electrodynamics in two dimensions, Ann. Phys. 68 (1971) 172.

[4] P. Becher, H. Joos, $1+1$ dimensional quantum electrodynamics as an illustration of the hypothetical structure of quark field theory, in: Proc. of the 5th meeting on fundamental physics. P.L. De Guevara et al. eds., Madrid, J. E. N., 1977.

[5] K.R. Ito, Two-dimensional massless quantum electrodynamics in the Landau-gauge formalism and the Higgs mechanism, Progr. Theor. Phys. 53 (1975) 817

[6] M. Lüscher, Bosonization in $2+1$ dimensions, Nucl. Phys. B326 (1989) 557

[7] D. Buchholz, On the manifestations of particles, in: Mathematical physics towards the 21st century. R.N. Sen, A. Gersten eds., Beer-Sheva, Ben Gurion University Press, 1994.

[8] D. Buchholz, R. Verch, Scaling algebras and renormalization group in algebraic quantum field theory. Preprint DESY 95-004, to appear in Rev. Math. Phys.

[9] S. Doplicher, J.E. Roberts, Why there is a field algebra with a compact gauge group describing the superselection structure in particle physics, Commun. Math. Phys. 131 (1990) 51

[10] R.F. Streater, A.S. Wightman, PCT, spin and statistics and all that, Benjamin, New York, 1964.

[11] J. Zinn-Justin, Quantum field theory and critical phenomena, Oxford, Clarendon-Press, 1989.

[12] D. Buchholz, K. Fredenhagen, Locality and the structure of particle states, Commun. Math. Phys. 84 (1982) 1. 
[13] D. Buchholz, The physical state space of quantum electrodynamics, Commun. Math. Phys. 85 (1982) 49

[14] D. Buchholz, Gauss' law and the infraparticle problem, Phys. Lett. B174 (1986) 331

[15] R. Haag, D. Kastler, An algebraic approach to quantum field theory, J. Math. Phys. 5 (1964) 848

[16] J. Langerholc, B. Schroer, Can current-operators determine a complete theory? Commun. Math. Phys. 4 (1967) 123

[17] J. Klauder, Poisson distributions and nontriviality of $\varphi^{4}$ theory, Phys. Rev. Lett. 73 (1994) 3051

[18] D. Buchholz, K. Fredenhagen, Dilations and interaction, J. Math. Phys. 18 (1977) 1107

[19] D. Buchholz, K. Fredenhagen, A note on the inverse scattering problem in quantum field theory, Commun. Math. Phys. 56 (1977) 91

[20] K. Baumann, All massless, scalar fields with trivial $S$-matrix are Wickpolynomials, Commun. Math. Phys. 86 (1982) 247

[21] D. Buchholz, On the structure of quantum fields with non-trivial interaction, in: Operator algebras, ideals, and their applications in Theoretical Physics, Proceedings Leipzig 1978. H. Baumgärtel et al. ed., Teubner, 1978.

[22] J. Magnen, V. Rivasseau, R. Sénéor, Construction of $Y M_{4}$ with an infrared cutoff, Commun. Math. Phys. 155 (1993) 325

[23] J. Fröhlich, G. Morchio, F. Strocchi, Charged sectors and scattering states in quantum electrodynamics, Ann. Phys. 119 (1979) 241

[24] R.F. Streater, I.F. Wilde, Fermion states of a boson field, Nucl. Phys. B24 (1970) 561

[25] G. Roepstorff, Coherent photon states and spectral condition, Commun. Math. Phys. 19 (1970) 301

[26] B. Schroer, Infrateilchen in der Quantenfeldtheorie, Fortschr. Phys. 11 (1963) 1

[27] D. Buchholz, M. Porrmann, U. Stein, Dirac versus Wigner. Towards a universal particle concept in local quantum field theory, Phys. Lett. B267 (1991) 377

[28] D. Buchholz, R. Verch, in preparation.

[29] M. Reed, B. Simon, Methods of modern mathematical physics II: Fourier analysis, self-adjointness, Academic Press, New York, 1975 